\title{
MICROBIOTA E BARREIRA INTESTINAL: IMPLICAÇÕES PARA OBESIDADE
}

\author{
MICROBIOTA AND INTESTINAL BARRIER: IMPLICATIONS FOR OBESITY
}

Clin Biomed Res. 2016;36(4):222-229

1 Programa de Pós-graduação em Nutrição Clínica e Estética, Instituto de Pesquisa e Gestão em Saúde (IPGS). Porto Alegre, RS, Brasil.

2 Serviço de Nutrição e Dietética, Hospital de Clínicas de Porto Alegre. Porto Alegre, RS, Brasil.

Autor correspondente:

Thais Ortiz Hammes

thaisortizhammes@gmail.com

Serviço de Nutrição e Dietética, Hospital

de Clínicas de Porto Alegre

Rua Ramiro Barcellos, 2350.

90035-903, Porto Alegre, RS, Brasil.

\section{Alynne Moniellen Oliveira' ${ }^{1}$, Thais Ortiz Hammes²}

\section{RESUMO}

A epidemia da obesidade é considerada um importante problema de saúde pública na sociedade ocidental, pois ela se relaciona a comorbidades como síndrome metabólica, diabetes mellitus e hipertensão. A microbiota intestinal pode contribuir para o desenvolvimento da obesidade através do aumento da extração energética dos componentes da dieta, da lipogênese, da permeabilidade intestinal e da endotoxemia, mediada especialmente pelos lipopolissacarídeos. Estudos têm demonstrado diferenças na composição da microbiota intestinal entre indivíduos obesos e magros. Ao que parece, o aumento na proporção de Firmicutes em relação a Bacteroidetes parece estar presente na obesidade, podendo ser alterado à medida que ocorre perda de peso. Assim, o objetivo deste estudo foi revisar a literatura acerca dos mecanismos que relacionam a microbiota e a barreira intestinal ao desenvolvimento ou agravamento da obesidade.

Palavras-chave: Obesidade; microbioma gastrointestinal; microbiota; permeabilidade intestinal

\section{ABSTRACT}

The epidemic of obesity is considered an important public health problem in the Western society and is related to comorbidities such as metabolic syndrome, diabetes mellitus, and hypertension. The intestinal microbiota may contribute to the development of obesity by increasing energy extraction from the dietary components, lipogenesis, intestinal permeability, and endotoxemia, especially mediated by lipopolysaccharides. Studies have demonstrated differences in composition of the intestinal microbiota between obese and lean individuals. Apparently, the increase in the proportion of Firmicutes in relation to Bacteroidetes seems to be present in obesity and can be changed during weight loss. The aim of this study was to review the mechanisms that relate microbiota and intestinal barrier to the development or worsening of obesity.

Keywords: Obesity; gastrointestinal microbiome; microbiota; intestinal permeability

A epidemia da obesidade é considerada um importante problema de saúde pública na sociedade ocidental, pois relaciona-se a comorbidades como síndrome metabólica, diabetes e hipertensão ${ }^{1}$. A Organização Mundial da Saúde (OMS) define a obesidade de acordo com o índice de massa corporal (IMC) e sua associação com a mortalidade, considerando o indivíduo com IMC maior ou igual a $30 \mathrm{~kg} / \mathrm{m}^{2}$ como obeso ${ }^{2}$. No Brasil, o excesso de peso atinge $50,1 \%$ da população adulta masculina e mais de $1 / 3$ dos meninos de 5 a 9 anos de idade ${ }^{3}$.

O peso corporal é determinado pela interação de fatores genéticos, culturais e ambientais ${ }^{4}$. Dentre estes, os fatores que predispõem à ingestão calórica excessiva e ao baixo gasto energético estão ligados à gênese da obesidade ${ }^{5}$. Um vez instalada, essa desordem metabólica traz consigo uma resposta inflamatória persistente e de baixa intensidade que afeta diversos órgãos e sistemas no corpo humano. Especificamente no trato gastrointestinal, a obesidade se relaciona a vários fatores: redução dos movimentos intestinais, 
supercrescimento bacteriano, disbiose, perda de integridade da barreira intestinal, translocação bacteriana, refluxo gastroesofágico e alterações no eixo cérebro-intestino ${ }^{6}$.

Recentemente, a interação entre microbiota e permeabilidade intestinal tem sido relacionada ao desenvolvimento não apenas da obesidade mas também de suas comorbidades ${ }^{7,8}$. A microbiota pode promover o fortalecimento da barreira intestinal e a proteção contra patógenos por meio da exclusão competitiva na ocupação de locais de fixação. Por outro lado, a quebra da integridade da barreira intestinal promove a entrada de moléculas potencialmente envolvidas no disparo de resposta inflamatória ${ }^{9}$. A evidência de que a composição da microbiota pode ser diferente em humanos magros e obesos reforçou a hipótese da sua influência na fisiopatologia da obesidade ${ }^{10}$.

Assim, o objetivo desta revisão é discutir os mecanismos que relacionam a permeabilidade intestinal e a microbiota intestinal ao desenvolvimento da obesidade e suas complicações.

\section{MICROBIOTA INTESTINAL E A REGULAÇÃO DO PESO CORPORAL DO HOSPEDEIRO}

O ser humano é amplamente habitado por micro-organismos. Estima-se que de 10 a 100 trilhões de bactérias, vírus e fungos sejam encontrados em nosso corpo, o que corresponde a 10 vezes o nosso número de células. Cerca de $70 \%$ dessas bactérias estão no intestino grosso e fazem parte da microbiota intestinal, um conjunto de micro-organismos residentes no intestino (tanto grosso como delgado) que coexistem em harmonia com o hospedeiro ${ }^{11,12}$. A relação entre hospedeiro e microbiota é mutualista. Assim, a interação entre eles pode proteger contra infecção de bactérias patogênicas, exercer função metabólica, com produção de uma ampla variedade de nutrientes, ter efeito imunomodulador e auxiliar a competência da barreira intestinal ${ }^{13}$.

A microbiota intestinal é formada pela presença de micro-organismos transientes e autóctones. Essa composição varia bastante dependendo da localização anatômica, da capacidade de adaptação dos micro-organismos e dos hábitos de vida do hospedeiro. Locais onde o trânsito intestinal é mais rápido, como o duodeno e jejuno, tendem a ter predomínio de microbiota transiente. Já o cólon, onde o trânsito intestinal pode ultrapassar 30 horas, possui uma microbiota autóctone bem definida. Aspectos relativos às bactérias, como habilidade de aderência ao muco, utilização de substratos e capacidade de competição com outras bactérias, definem o perfil de micro-organismos que colonizam cada indivíduo. Assim, os grupos bacterianos mais abundantes na microbiota intestinal de humanos são os Firmicutes, Bacteroidetes e Actinobacteria ${ }^{14}$.

A composição da microbiota intestinal varia ao longo da vida. Nos primeiros 3 anos de idade, os micro-organismos que habitam o intestino dependem essencialmente da interação da criança com o ambiente ${ }^{15}$. A partir dessa idade, a microbiota intestinal apresenta semelhança importante com aquela observada nos indivíduos adultos. Na fase adulta, a composição da microbiota é relativamente estável, sendo difícil a colonização por um micro-organismo não autóctone ${ }^{16}$. Nessa faixa etária, em geral há uma proporção maior de Firmicutes em relação a Bacteroidetes $^{17}$.

Ley et al. ${ }^{18,19}$ observaram que indivíduos obesos apresentam uma razão Firmicutes/Bacteroidetes maior que a encontrada em indivíduos magros. Em estudo em seres humanos, indivíduos obesos foram submetidos, durante 52 semanas, a um programa de emagrecimento com dois tipos diferentes de dieta: uma com restrição de lipídeos e outra com restrição de carboidratos ${ }^{19}$. Como resultado, observou-se que o emagrecimento, independentemente do tipo de dieta, promoveu alteração da microbiota intestinal, de forma que esta tornou-se semelhante à microbiota de indivíduos magros, reforçando a relação entre o peso corporal e a microbiota intestinal ${ }^{19}$.

A ingestão alimentar do hospedeiro é considerada o principal substrato para o crescimento da microbiota e também afeta diretamente sua composição ${ }^{20}$. Dietas ricas em proteína podem propiciar a produção de aminas, fenóis e amônia pelas proteases da microbiota intestinal. Esses compostos estão relacionados a um efeito pró-carcinogênico em diversos tecidos, especialmente no cólon. Ratos alimentados com dieta contendo $15 \%$ de proteína de baixa digestibilidade, quando expostos ao carcinogênico azoximetano, apresentaram maior fermentação proteica, maiores níveis urinários de $p$-cresol e aumento na incidência de adenocarcinoma intestinal em comparação aos animais que receberam dieta contendo $10 \%$ de amido resistente ${ }^{21}$. Embora a extrapolação de dados de modelos murinos possa ser limitada, evidências semelhantes também foram observadas em estudos em humanos ${ }^{22-24}$. Nesse sentido, determinados grupos bacterianos (E. coli, Klebsiella pneumoniae, Clostridium spp.) podem produzir sulfeto de hidrogênio, um gás citotóxico para a mucosa intestinal, quando da presença de alimentos ricos em enxofre, como bebidas alcoólicas, carnes, leites, ovos, vegetais crucíferos e frutos do $\operatorname{mar}^{25,26}$.

Proteases bacterianas apresentam atividade enzimática ótima em $\mathrm{pH}$ neutro ${ }^{25,26}$. Assim, a presença de bactérias produtoras de ácido poderia reduzir o $\mathrm{pH}$ luminal e diminuir a atividade proteolítica dessas 
enzimas ${ }^{25,26}$. De fato, indivíduos saudáveis tratados por 4 semanas com Lactobacillus casei Shirota apresentaram redução de atividade proteolítica colônica (excreção urinária de $p$-cresol) e aumento no conteúdo fecal de Bifidobacteria ${ }^{22}$. Já o consumo de prebióticos durante o mesmo período promoveu não apenas a redução da excreção urinária de fenol como também a diminuição da eliminação de nitrogênio na urina ${ }^{22}$. Embora importantes, esses resultados devem ser analisados com cautela, uma vez que os indivíduos estudados $(n=10)$ não foram controlados quanto ao consumo de proteínas na dieta.

Os açúcares também têm papel fundamental sobre a composição microbiana do intestino. Sacarídeos não absorvidos pelo intestino são hidrolisados por bactérias intestinais produzindo gases e ácidos graxos de cadeia curta (AGCC). Os principais AGCC encontrados no colón de seres humanos são acetato, propionato e butirato, em uma razão próxima a 1:1:327. Noventa por cento dos AGCC são absorvidos pelos colonócitos; o butirato é quase totalmente utilizado como fonte energética nas células intestinais e o restante atinge a circulação portal. Por esse motivo, AGCC podem fornecer calorias adicionais quando oxidados pelo hospedeiro e favorecer o ganho de peso na forma de gordura corporal28.

Estudo em modelo murino mostrou que animais obesos possuem microbiota com alta capacidade sacarolítica, uma vez que expressam várias enzimas responsáveis pela quebra de polissacarídeos não digeríveis da dieta ${ }^{29}$. Como resultado, a produção de AGCC é maior e o conteúdo energético excretado nas fezes é reduzido, já que a digestão dos polissacarídeos gera monossacarídeos absorvíveis pelo epitélio intestinal. Isso sugere que, dependendo da microbiota, o hospedeiro pode extrair quantidades variadas de calorias da dieta. De fato, mulheres obesas $(n=42)$ apresentam níveis fecais maiores de AGCC quando comparadas a mulheres eutróficas ${ }^{30}$. No entanto, as calorias adicionais fornecidas pela fermentação de carboidratos não digeridos podem não ser suficientes para produzir mudanças significativas no peso. Um dos argumentos que suportam essa hipótese é o de que o consumo de uma dieta rica em fibras, que pode aumentar a produção de AGCC, normalmente ajuda a reduzir o peso corporal ${ }^{31,32}$.

Os AGCC também atuam como mediadores químicos que transmitem as informações do lúmen intestinal - produzidas pela interação entre microbiota e hospedeiro - para o restante do corpo através de receptores localizados no epitélio intestinal ${ }^{27}$. Dessa forma, AGCC podem regular o metabolismo energético, a imunidade e a expansão do tecido adiposo. Isso se deve à sua capacidade de ativar receptores implicados no mecanismo de inibição da lipólise e de diferenciação dos adipócitos ${ }^{33}$. Outra forma de atuação dos AGCC é através da modulação dos hormônios envolvidos no controle do apetite. Altas concentrações de AGCC parecem induzir a proliferação de células $L$ intestinais e, consequentemente, aumentar a secreção de seus produtos.

As células $L$ são células enteroendócrinas localizadas no íleo e no cólon, produtoras do peptídeo YY (PYY) e do peptídeo semelhante ao glucagon (glucagon-like peptide, GLP-1) ${ }^{27}$. O PYY é um hormônio anorexígeno capaz de reduzir a velocidade do trânsito intestinal. Já o GLP-1 é um hormônio que estimula a liberação de insulina e possui um efeito antidiabético. As células $L$ também apresentam abundante expressão de receptores de ácidos graxos livres (free fatty acids) do tipo 2 (FFA2). Estes receptores são ativados por AGCCs, promovendo supressão da inflamação e secreção de GLP-1 e PYY 27,34 . Hadav et al. ${ }^{35}$ observaram que animais expostos a dieta hiperlipídica tratados com probiótico VSL\#3 apresentavam ganho de peso e ingestão alimentar inferiores aos dos animais que recebiam dieta rica em gordura porém sem tratamento com probiótico. Nesse mesmo estudo, foi observado que animais tratados com probiótico tinham aumento de butirato nas fezes ${ }^{35}$. Em estudo in vitro, linhagens de células $\mathrm{L}$ humanas $(\mathrm{NCl}-\mathrm{H} 716)$ aumentaram a produção de GLP-1 quando expostas a doses de 1-2 mM de butirato ${ }^{35}$. Por outro lado, o aumento de butirato para 5 e $10 \mathrm{mM}$ promoveu redução progressiva da secreção de GLP-1, mostrando uma possível relação de dose-resposta entre a presença de AGCC no lúmen e a produção de hormônios da saciedade ${ }^{35}$.

A microbiota intestinal também pode atuar na regulação de genes responsáveis por modular o gasto e o armazenamento de energia ${ }^{31}$. Um dos mecanismos associados a esse efeito pode estar relacionado à inibição do fator de adipócitos induzido por jejum (fasting-induced adipose factor, FIAF) pela microbiota intestinal. O FIAF é um inibidor da lípase lipoproteica (LPL) produzido pelo tecido adiposo, fígado e intestino ${ }^{36}$. A LPL é uma enzima que hidrolisa triglicerídeos e estimula o seu armazenamento nos adipócitos. A colonização intestinal de animais livres de germes pode induzir ao aumento da atividade da LPL através de supressão de FIAF $^{32}$. Camundongos livres de germes apresentaram aumento do peso corporal, das concentrações de triglicerídeos hepáticos e da expressão de proteínas responsáveis pela adipogênese 14 dias após a colonização intestinal ${ }^{36}$. Animais recolonizados também tiveram redução da expressão de FIAF intestinal e aumento na expressão de LPL em diversos tecidos ${ }^{36}$. Assim, a inibição da 
expressão de FIAF pela presença de microbiota específica parece permitir o aumento da atividade da LPL e o acúmulo de triglicérides no tecido adiposo ${ }^{28}$.

Em seres humanos, o transplante de microbiota também parece trazer impacto sobre o metabolismo. Um ensaio clínico randomizado avaliou o efeito do transplante da microbiota de doadores magros (IMC $\left.<23 \mathrm{~kg} / \mathrm{m}^{2}\right)$, do sexo masculino, em paciente com síndrome metabólica $(n=9)^{37}$. Seis semanas após o procedimento, foi observado aumento significativo na sensibilidade à insulina (de 26,2 para $45,3 \mu \mathrm{mol} / \mathrm{kg} / \mathrm{min} ; \mathrm{p}<0,05)$ e na diversidade de espécies da microbiota em pacientes que receberam transplante fecal. A quantidade de AGCC nas fezes também foi significativamente menor após o transplante. De forma semelhante, Alang e Kelly ${ }^{38}$ relataram o caso de uma paciente com peso estável que apresentou súbito aumento de peso e do apetite após ser submetida ao transplante de fezes para o tratamento de infecção por Clostridium difficile. Nesse caso, o doador apresentava sobrepeso e histórico de aumento de peso no período próximo ao transplante. Esse relato reforça o papel da microbiota como um possível indutor de alterações metabólicas envolvidas na regulação do peso corporal.

Apesar do exposto, os mecanismos que relacionam a microbiota intestinal à homeostase do peso corporal são complexos. Embora ainda existam dúvidas sobre qual o perfil de micro-organismos relacionados à obesidade, muito se conhece sobre sua influência na saúde do hospedeiro. O que se sabe até o momento é que a microbiota pode afetar o balanço de energia influenciando a eficiência energética dos nutrientes da dieta ou atuando como modulador gênico sobre as rotas que regulam o gasto energético.

\section{A BARREIRA INTESTINAL E A OBESIDADE}

O intestino está constantemente sendo exposto a partículas potencialmente patogênicas, tais como antígenos dietéticos e micro-organismos. É interessante notar que, da mesma forma que o sistema imune intestinal é exímio em sua função de proteção, combatendo os invasores, também é tolerante aos micro-organismos autóctones do hospedeiro ${ }^{13,39}$. Nesse sentido, é importante que as barreiras física e imunológica sejam complementares.

O epitélio intestinal funciona como a barreira física que restringe a entrada de moléculas antigênicas, ao mesmo tempo em que convive em equilíbrio com a exposição a uma quantidade enorme de componentes dietéticos e microbianos ${ }^{40}$. A capacidade de contenção da barreira intestinal é considerada dinâmica, pois pode ser afetada por diversos fatores como doenças, medicamentos, hormônios, citocinas e toxinas exógenas $^{17,41}$. O grau de abalo da competência da barreira intestinal é variável e sua duração dependente do tipo e da presença do estímulo agressor. Várias células compõem o sistema de defesa intestinal, que apresenta como característica uma alta taxa de renovação epitelial (Quadro 1).

Já a barreira imunológica intestinal é composta pelo tecido linfoide associado ao intestino (gut-associated lymphoid tissue, GALT) e por imunoglobulinas. O GALT abriga cerca de $30 \%$ de todos os linfócitos do corpo, onde são encontradas as placas de Peyer, principalmente no íleo distal. As placas de Peyer são recobertas por células $M$, as quais capturam antígenos do lúmen e os entregam para que as células dendríticas façam a apresentação de antígeno ${ }^{39,42}$. As imunoglobulinas luminais também são responsáveis

Quadro 1: Células que compõem a barreira intestinal.

\begin{tabular}{|c|l|}
\hline \multicolumn{1}{|c|}{ Células } & \multicolumn{1}{c|}{ Função } \\
\hline Enterócitos & $\begin{array}{l}\text { Representam 80\% de todas as células epiteliais no intestino. Possuem junções } \\
\text { apertadas bastante eficientes, o que auxilia a separação entre conteúdos luminais } \\
\text { e o interior do epitélio. Podem atuar como células apresentadoras de antígenos, } \\
\text { expressando moléculas MHC (major histocompatibility complex) classe Il e receptores } \\
\text { do tipo Tol/40. }\end{array}$ \\
\hline Células caliciformes & $\begin{array}{l}\text { Responsáveis pela produção de mucina intraluminal. Mucinas, eletrólitos e proteoglicanos } \\
\text { formam o muco que reveste a superfície das vilosidades intestinais e mantêm os } \\
\text { vários componentes patogênicos separados do epitélio. O muco também é capaz } \\
\text { de aprisionar antígenos e bactérias, os quais podem ser hidrolisados - tornando-se } \\
\text { menos alergênicos - ou ser excretados com auxílio dos movimentos peristálticos } \\
\text { intestinais }\end{array}$ \\
\hline Células.
\end{tabular}


pela captação de antígenos ou micro-organismos e podem impedir a ligação desses antígenos com receptores das células do hospedeiro, reduzindo a resposta inflamatória ${ }^{39,40}$. A imunoglobulina $\mathrm{A}(\lg \mathrm{A})$ secretória é a principal representante da imunidade humoral do intestino, sendo que sua produção pode ser afetada pela microbiota comensal. Experimentos com animais livres de germes evidenciaram baixos níveis de lgA, hipoplasia das placas de Peyer e uma precária formação linfonodal - eventos que foram revertidos após a inoculação de bactérias comensais ${ }^{13}$.

Componentes microbianos oriundos do intestino podem induzir resposta inflamatória no hospedeiro, pois o sistema imune humano é capaz de reconhecer padrões moleculares associados aos patógenos (pathogen-associated molecular patterns, PAMPs) como invasores. Esse reconhecimento promove uma sinalização de alerta ao organismo e atua principalmente via receptores do tipo Toll (Toll-like receptors, TLR). Assim, os TLR têm como função essencial sinalizar a presença de patógenos ao organismo. No entanto, nem todos os TLR (TLR 2, $4,5,6,7$, e 9) respondem da mesma forma, pois alguns subtipos podem até mesmo aumentar a ligação das junções. Outros subtipos estão localizados em regiões onde as bactérias somente alcançariam se estivessem invadindo a mucosa - o que exigiria resposta rápida e eficaz ${ }^{39}$.

Os lipopolissacarídeos (LPS) são reconhecidos como PAMPs pelos TLR do hospedeiro e são capazes de induzir resposta inflamatória de ataque assim que são percebidos. Os LPS são componentes da parede celular de bactérias gram-negativas com capacidade de induzir a resposta inflamatória e danificar a barreira intestinal. São considerados endotoxinas, uma vez que podem ativar inflamação crônica em doses baixas. O lúmen intestinal é um reservatório de LPS e sua entrada para a circulação pode ocorrer por meio de duas vias: a difusão direta através do aumento da permeabilidade intestinal (devido ao afrouxamento do espaço paracelular intestinal) ou por meio da absorção e incorporação de LPS na estrutura dos quilomícrons ${ }^{31}$.

A endotoxemia resultante do aumento dos níveis circulantes de LPS pode afetar tecidos alvo, como tecido adiposo, fígado e endotélio. Animais livres de germes não possuem níveis elevados de LPS e sua resistência ao desenvolvimento de obesidade pode ser parcialmente relacionada a um estímulo inflamatório reduzido. É importante lembrar que a microbiota intestinal é também uma fonte de muitas outras moléculas inflamatórias, tais como peptidoglicano, lipoproteínas e flagelina, que também podem ligar-se aos $\operatorname{TLR}^{31}$.
A ingestão crônica de dieta rica em lipídeos leva à mudança no perfil da microbiota intestinal e pode aumentar significativamente a concentração plasmática de LPS ${ }^{43}$. Camundongos expostos a uma dieta com $72 \%$ de lipídeos apresentaram aumento nos níveis circulantes de LPS comparável ao de animais sem manipulação dietética que receberam injeções subcutâneas contendo $300 \mathrm{~g} / \mathrm{kg} / \mathrm{dia}$ de LPS $^{44}$. Neste estudo, o grupo que recebeu dieta hiperlipídica ingeriu quase o dobro de calorias que os demais grupos. No entanto, o ganho de peso dos animais que tiveram apenas injeção de LPS foi semelhante ao daqueles expostos à dieta hiperlipídica. Metabolicamente, ambos os grupos apresentaram aumento nos valores de glicemia de jejum, insulina plasmática, citocinas inflamatórias e peso corporal, às custas principalmente do aumento do tecido adiposo ${ }^{44}$. Esses resultados demonstram que a endotoxemia isoladamente pode estar relacionada ao desenvolvimento da obesidade e suas complicações, colocando novamente a microbiota como possível agente causal ${ }^{44,45}$.

A inflamação também pode reduzir a integridade intestinal por lesar a barreira mecânica e permitir a permeação de moléculas através da parede intestinal. Animais submetidos a dieta hiperlipídica apresentam aumento da permeabilidade intestinal e redução da expressão de zonulina e ocludina no epitélio do intestino ${ }^{45}$. O papel da microbiota na competência da barreira intestinal é de tal forma importante que animais expostos à dieta hiperlipídica, quando cotratados com antibiótico, apresentam redução na permeabilidade intestinal a níveis próximos aos observados nos controles $^{45}$. De forma semelhante, mulheres obesas apresentam aumento da permeabilidade intestinal, avaliada pelo percentual de excreção de lactulose, em comparação a mulheres não obesas ${ }^{46}$. Outro estudo encontrou níveis circulantes de zonulina maiores em indivíduos obesos em relação aos valores observados em não obesos, sendo esses valores correlacionados positivamente com o IMC ${ }^{47}$. Esses resultados apontam uma relação importante entre permeabilidade intestinal e obesidade, no entanto, não permitem estabelecer causalidade. Assim, tanto a obesidade pode induzir o aumento da permeabilidade intestinal quanto esta pode levar ao desenvolvimento/agravamento da obesidade.

Em relação à permeabilidade intestinal, sabe-se que, quanto maior o dano à parede intestinal, maior será o tamanho da molécula que poderá atravessar a barreira intestinal e penetrar na corrente sanguínea. Essas moléculas, ao atravessarem a barreira intestinal, iniciam uma resposta imune proporcional à antigenicidade e à frequência de exposição ao componente lesivo. A quebra da integridade da 
barreira intestinal e o aumento da permeação têm sido associados aos mecanismos etiopatogênicos comuns a várias doenças de caráter inflamatório como o diabetes e a obesidade ${ }^{9,45}$.

\section{CONCLUSÃO}

O exato mecanismo pelo qual a microbiota e a permeabilidade intestinal contribuem para o ganho de peso permanece incerto. No entanto, acredita-se que a microbiota intestinal pode contribuir para o desenvolvimento da obesidade através do aumento da extração energética dos componentes da dieta, da lipogênese, da permeabilidade intestinal e da endotoxemia, mediada especialmente pela LPS ${ }^{31}$.

Mais estudos são necessários para determinar o real impacto desses mecanismos no desenvolvimento da obesidade. Assim, o conhecimento da microecologia intestinal e de suas vias de regulação do peso corporal pode ajudar na identificação de novos alvos terapêuticos.

\section{REFERÊNCIAS}

1. Tremaroli V, Backhed F. Functional interactions between the gut microbiota and host metabolism. Nature. 2012 13;489:242-9. http:// dx.doi.org/10.1038/nature11552.

2. World Health Organization (WHO). Obesity: preventing and managing the global epidemic. Report of a WHO consultation. World Health Organ Tech Rep Ser. 2000;894:i-xii, 1-253. PMid:11234459.

3. Instituto Brasileiro de Geografia e Estatística (IBGE). Pesquisa de Orçamentos Familiares 2008-2009: antropometria e estado nutricional de crianças, adolescentes e adultos no Brasil In: Instituto Brasileiro de Geografia e Estatística (IBGE). Estatística. Rio de Janeiro: IBGE; 2010.

4. Hammes TO, Costa CS, Rohden F, Margis R, de Almeida JC, Padoin $A V$, et al. Parallel down-regulation of FOX01, PPARy and adiponectin mRNA expression in visceral adipose tissue of class III obese individuals. Obes Facts. 2012;5(3):452-9. PMid:22797373. http://dx.doi. org/10.1159/000339574.

5. Pi-Sunyer FX. The obesity epidemic: pathophysiology and consequences of obesity. Obes Res. 2002;10(Suppl 2):97S-104S. PMid:12490658. http:// dx.doi.org/10.1038/oby.2002.202.

6. Teixeira TF, Collado MC, Ferreira CL, Bressan J, Peluzio MC. Potential mechanisms for the emerging link between obesity and increased intestinal permeability. Nutr Res. 2012;32(9):637-47. PMid:23084636. http://dx.doi.org/10.1016/j. nutres.2012.07.003.

7. Bischoff SC, Barbara G, Buurman W, Ockhuizen T, Schulzke JD, Serino $\mathrm{M}$, et al. Intestinal permeability--a new target for disease prevention and therapy. BMC Gastroenterol. 2014;14(1):189. PMid:25407511. http://dx.doi.org/10.1186/s12876-0140189-7.

8. Perpétuo JP, Wilasco MI, Schneider AC. The role of intestinal microbiota in energetic metabolism: new perspectives in combating obesity. Clin Biomed Res. 2015;35(4):1969. http://dx.doi.org/10.4322/23579730.60358

9. Luoto R, Collado MC, Salminen $\mathrm{S}$, Isolauri $\mathrm{E}$. Reshaping the gut microbiota at an early age: functional impact on obesity risk? Ann Nutr Metab. 2013;63(Suppl 2):17-26. PMid:24217033. http://dx.doi. org/10.1159/000354896.

10. Tsukumo DM, Carvalho BM, CarvalhoFilho MA, Saad MJ. Translational research into gut microbiota: new horizons in obesity treatment. Arq Bras Endocrinol Metabol. 2009;53(2):139-44. PMid:19466205. http://dx.doi.org/10.1590/S000427302009000200004.

11. Cani PD, Delzenne NM. The gut microbiome as therapeutic target. Pharmacol Ther. 2011;130(2):202-12. PMid:21295072. http://dx.doi.org/10.1016/j. pharmthera.2011.01.012.

12. Sekirov I, Russell SL, Antunes LC, Finlay BB. Gut microbiota in health and disease. Physiol Rev. 2010;90(3):859-904. PMid:20664075. http://dx.doi.org/10.1152/ physrev.00045.2009.

13. Yu LC, Wang JT, Wei SC, Ni YH. Host-microbial interactions and regulation of intestinal epithelial barrier function: from physiology to pathology. World J Gastrointest Pathophysiol. 2012;3(1):27-43. PMid:22368784. http://dx.doi.org/10.4291/wjgp. v3.i1.27.

14. Million M, Lagier JC, Yahav D, Paul M. Gut bacterial microbiota and obesity. Clin Microbiol Infect. 2013;19(4):30513. PMid:23452229. http://dx.doi. org/10.1111/1469-0691.12172.

15. Arrieta MC, Stiemsma LT, Amenyogbe $\mathrm{N}$, Brown EM, Finlay B. The intestinal microbiome in early life: health and disease. Front Immunol. 2014;5:427. PMid:25250028. http://dx.doi. org/10.3389/fimmu.2014.00427.

16. Adlerberth I, Cerquetti M, Poilane I, Wold A, Collignon A. Mechanisms of colonisation and colonisation resistance of the digestive tract part 2: bacteria/bacteria interactions. Microb Ecol Health Dis. 2000;12:223-39. http:// dx.doi.org/10.1080/0891060007500604 86.

17. Cresci GA, Bawden E. Gut microbiome: what we do and don't know. Nutr Clin Pract. 2015;30(6):73446. PMid:26449893. http://dx.doi. org/10.1177/0884533615609899.

18. Ley RE, Backhed F, Turnbaugh P, Lozupone CA, Knight RD, Gordon Jl. Obesity alters gut microbial ecology. Proc Natl Acad Sci USA. 2005;102(31):110705. PMid:16033867. http://dx.doi. org/10.1073/pnas.0504978102.

19. Ley RE, Turnbaugh PJ, Klein S, Gordon JI. Microbial ecology: human gut microbes associated with obesity Nature. 2006;444(7122):10223. PMid:17183309. http://dx.doi. org/10.1038/4441022a.

20. Devkota S, Chang EB. Nutrition, microbiomes, and intestinal inflammation. Curr Opin Gastroenterol. 2013;29(6):603-7. PMid:24100722. http://dx.doi.org/10.1097/ MOG.0b013e328365d38f. 
21. Le Leu RK, Brown IL, Hu Y, Morita T, Esterman A, Young GP. Effect of dietary resistant starch and protein on colonic fermentation and intestinal tumourigenesis in rats. Carcinogenesis. 2007;28(2):2405. PMid:17166881. http://dx.doi. org/10.1093/carcin/bgl245.

22. De Preter V, Vanhoutte T, Huys G, Swings J, De Vuyst L, Rutgeerts $P$, et al. Effects of Lactobacillus casei Shirota, Bifidobacterium breve, and oligofructose-enriched inulin on colonic nitrogen-protein metabolism in healthy humans. Am J Physiol Gastrointest Liver Physiol. 2007;292(1):G358-68. PMid:16990449. http://dx.doi. org/10.1152/ajpgi.00052.2006.

23. Birkett A, Muir J, Phillips J, Jones G, O'Dea K. Resistant starch lowers fecal concentrations of ammonia and phenols in humans. Am J Clin Nutr. 1996;63(5):766-72. PMid:8615362.

24. Muir JG, Yeow EG, Keogh J, Pizzey C, Bird AR, Sharpe K, et al. Combining wheat bran with resistant starch has more beneficial effects on fecal indexes than does wheat bran alone. Am J Clin Nutr. 2004;79(6):1020-8. PMid:15159232.

25. Bernalier-Donadille A. Main metabolic funtions of the human intestinal microflora. In: Rambaud J-C, Buts J-P, Corthier G, Flourie B, editors. Gut microflora: digestive physiology and pathology. Montrouge: John Libbey Eurotext; 2006. p. 71-80.

26. Yao CK, Muir JG, Gibson PR. Review article: insights into colonic protein fermentation, its modulation and potential health implications. Aliment Pharmacol Ther. 2016;43(2):18196. PMid:26527169. http://dx.doi. org/10.1111/apt.13456.

27. Kaji I, Karaki S, Kuwahara A. Shortchain fatty acid receptor and its contribution to glucagon-like peptide-1 release. Digestion. 2014;89(1):316. PMid:24458110. http://dx.doi. org/10.1159/000356211.

28. Moreira APB, Teixeira TFS, Peluzio MCG, Alfenas RCG. Gut microbiota and the development of obesity. Nutr Hosp. 2012;27(5):1408-14. PMid:23478685.

29. Turnbaugh PJ, Ley RE, Mahowald MA, Magrini V, Mardis ER, Gordon JI. An obesity-associated gut microbiome with increased capacity for energy harvest.
Nature. 2006;444(7122):102731. PMid:17183312. http://dx.doi. org/10.1038/nature05414.

30. Fernandes J, Su W, RahatRozenbloom S, Wolever TM, Comelli EM. Adiposity, gut microbiota and faecal short chain fatty acids are linked in adult humans. Nutr Diabetes. 2014;4:e121. http://dx.doi. org/10.1038/nutd.2014.23.

31. Moreira APB, Teixeira TFS, Peluzio MCG, Alfenas RCG. Gut microbiota and the development of obesity. Nutr Hosp. 2012;27(5):1408-14. PMid:23478685.

32. Bäckhed F, Manchester JK, Semenkovich CF, Gordon JI. Mechanisms underlying the resistance to diet-induced obesity in germfree mice. Proc Natl Acad Sci USA. 2007;104(3):979-84. PMid:17210919. http://dx.doi.org/10.1073/ pnas.0605374104.

33. Delzenne NM, Cani PD. Interaction between obesity and the gut microbiota: relevance in nutrition. Annu Rev Nutr. 2011;31(1):1531. PMid:21568707. http:// dx.doi.org/10.1146/annurevnutr-072610-145146.

34. Psichas A, Sleeth ML, Murphy KG, Brooks L, Bewick GA, Hanyaloglu $A C$, et al. The short chain fatty acid propionate stimulates GLP-1 and PYY secretion via free fatty acid receptor 2 in rodents. Int $J$ Obes. 2015;39(3):424-9. PMid:25109781. http://dx.doi.org/10.1038/ijo.2014.153.

35. Yadav H, Lee JH, Lloyd J, Walter P, Rane SG. Beneficial metabolic effects of a probiotic via butyrateinduced GLP-1 hormone secretion. J Biol Chem. 2013;288(35):2508897. PMid:23836895. http://dx.doi. org/10.1074/jbc.M113.452516.

36. Backhed F, Ding H, Wang T, Hooper LV, Koh GY, Nagy A, et al. The gut microbiota as an environmental factor that regulates fat storage. Proc Natl Acad Sci USA. 2004;101(44):1571823. PMid:15505215. http://dx.doi. org/10.1073/pnas.0407076101.

37. Vrieze A, Van Nood E, Holleman F, Salojarvi J, Kootte RS, Bartelsman JF, et al. Transfer of intestinal microbiota from lean donors increases insulin sensitivity in individuals with metabolic syndrome. Gastroenterology. 2012;143(4):9136.e7. http://dx.doi.org/10.1053/j. gastro.2012.06.031.
38. Alang N, Kelly CR. Weight gain after fecal microbiota transplantation. Open Forum Infect Dis. 2015;2(1):ofv004. PMid:26034755. http://dx.doi. org/10.1093/ofid/ofv004.

39. Abbas AK, Lichtman AHH, Pillai S. Regional immunity: specialized immune responses in epithelial and immune privileged tissues In: Abbas AK, Lichtman AHH, Pillai S, editores. Cellular and molecular immunology. 7th ed. Philadelphia: Saunders; 2011. p. 295-307.

40. Ramiro-Puig E, Pérez-Cano FJ, Castellote C, Franch A, Castell M. The bowel: a key component of the immune system. Rev Esp Enferm Dig. 2008;100(1):29-34. PMid:18358058.

41. Natividad JM, Verdu EF. Modulation of intestinal barrier by intestinal microbiota: pathological and therapeutic implications. Pharmacol Res. 2013;69(1):4251. PMid:23089410. http://dx.doi. org/10.1016/j.phrs.2012.10.007.

42. Acheson DW, Luccioli S. Microbial-gut interactions in health and disease. Mucosal immune responses. Best Pract Res Clin Gastroenterol. 2004;18(2):387-404. PMid:15123077. http://dx.doi.org/10.1016/j. bpg.2003.11.002.

43. Raybould HE. Gut microbiota, epithelial function and derangements in obesity. J Physiol. 2012;590(3):4416. PMid:22183718. http://dx.doi. org/10.1113/jphysiol.2011.222133.

44. Cani PD, Amar J, Iglesias MA, Poggi M, Knauf C, Bastelica D, et al. Metabolic endotoxemia initiates obesity and insulin resistance. Diabetes. 2007;56(7):1761-72. PMid:17456850. http://dx.doi. org/10.2337/db06-1491.

45. Cani PD, Bibiloni R, Knauf $C$, Waget A, Neyrinck AM, Delzenne NM, et al. Changes in gut microbiota control metabolic endotoxemiainduced inflammation in high-fat diet-induced obesity and diabetes in mice. Diabetes. 2008;57(6):147081. PMid:18305141. http://dx.doi. org/10.2337/db07-1403.

46. Teixeira TF, Souza NC, Chiarello PG, Franceschini SC, Bressan J, Ferreira $\mathrm{CL}$, et al. Intestinal permeability parameters in obese patients are correlated with metabolic syndrome risk factors. Clin Nutr. 2012;31(5):73540. PMid:22444236. http://dx.doi. org/10.1016/j.clnu.2012.02.009. 
47. Moreno-Navarrete JM, Sabater M, Ortega F, Ricart W, Fernandez-Real JM. Circulating zonulin, a marker of intestinal permeability, is increased in association with obesity-associated insulin resistance. PLoS One. 2012;7(5):e37160. PMid:22629362. http://dx.doi.org/10.1371/journal. pone. 0037160

Recebido: Set 08, 2016 Aceito: Nov 23, 2016 